\title{
Additional Effect of Coronary High-Intensity Plaque on T1-Weighted Magnetic Resonance Imaging With Circulating Malondialdehyde-Modified Low-Density Lipoprotein on Cardiac Events
}

\author{
Daigo Hiraya, MD; Akira Sato, MD; Tomoya Hoshi, MD, PhD; \\ Shunsuke Sakai, MD; Hiroaki Watabe, MD, PhD; Masaki Ieda, MD, PhD
}

Background: Although elevated levels of oxidized low-density lipoprotein (LDL) could play a critical role in vulnerable plaque, there are no studies that have compared coronary high-intensity plaque (HIP) and circulating malondialdehyde-modified (MDA)-LDL levels for the prediction of cardiac events.

Methods and Results: A total of 139 patients with coronary artery stenosis (>70\%) were examined with non-contrast T1-weighted magnetic resonance imaging (MRI) (HIP: $n=64$, non-HIP: $n=75)$. Scheduled percutaneous coronary intervention (PCl) for culprit lesions was performed within $48 \mathrm{~h}$ after MRI. HIP was defined as a signal intensity of coronary plaque to cardiac muscle ratio (PMR) 21.4. We evaluated the subsequent major adverse cardiac events (MACE) during the follow-up period (5.6 11.3 years). MDA-LDL levels were independently associated with the presence of HIP $(\mathrm{P}<0.0001)$. The incidence of MACE was $15 \%$, and it was significantly higher in patients with HIP $(27 \%)$ than in those without HIP $(5 \% ; P=0.011)$. Cox proportional hazard analysis showed MDA-LDL levels $(P=0.007)$ and PMR $(P=0.016)$ were significantly associated with MACE. For MACE prediction, $C$-statistic values for MDA-LDL, PMR, and PMR+MDA-LDL were 0.724, 0.791, and 0.800, respectively. Compared with MDA-LDL alone, the addition of PMR to MDA-LDL increased net reclassification improvement by $0.78(\mathrm{P}=0.012)$.

Conclusions: MDA-LDL levels might be associated with the presence of HIP in patients with coronary artery disease. Furthermore, adding PMR to MDA-LDL levels markedly improved prediction of subsequent MACE after PCI.

Key Words: Cardiac events; Coronary high-intensity plaque; Magnetic resonance imaging; Malondialdehyde-modified low-density lipoprotein

$\mathbf{T}$ he detection of vulnerable or high-risk atherosclerotic plaques is important in the prevention of acute coronary syndrome (ACS); however, there are a limited number of methods that are sufficiently sensitive to specifically identify such plaques. Vulnerable plaque has a large lipid-rich necrotic core, a thin-fibrotic cap, and numerous inflammatory cells. ${ }^{1}$ Non-contrast T1-weighted imaging (T1WI) using cardiac magnetic resonance (CMR) non-invasively highlights intraplaque components with short T1 having a high signal intensity. A necrotic core with intraplaque hemorrhage (IPH) or thrombus gives rise to a short T1 signal. Therefore, high-intensity plaque (HIP) detected on T1WI is considered to be vulnerable or highrisk coronary plaque, making it possible to detect the culprit lesion in patients with unstable angina. ${ }^{2,3}$ Noguchi et al reported that the presence of both HIP and a coronary plaque to myocardium signal intensity ratio $(\mathrm{PMR}) \geq 1.4$

\section{Editorial p 2040}

were risk factors for future coronary events among patients with coronary artery disease (CAD). ${ }^{4}$ We previously demonstrated that the presence of coronary HIP is associated with myocardial injury after percutaneous coronary intervention (PCI). ${ }^{5}$

Oxidatively modified low-density lipoprotein (OxLDL) plays an important role in the development of atherosclerosis because its uptake by macrophages and smooth muscle cells leads to the formation of foam cells, which is a critical step in the evolution of the pathological state of vulnerable plaque. OxLDL is incorporated into macrophages by scavenger receptors and modulates various vascular functions, including inhibition of nitric oxide generation, induction of endothelial apoptosis, proliferation of smooth muscle

Received March 11, 2021; revised manuscript received June 6, 2021; accepted June 8, 2021; J-STAGE Advance Publication released online July 17, 2021 Time for primary review: 14 days

Department of Cardiology, Faculty of Medicine, University of Tsukuba, Tsukuba, Japan

Mailing address: Akira Sato, MD, Department of Cardiology, Faculty of Medicine, University of Tsukuba, 1-1-1 Tennodai, Tsukuba 305-8575, Japan. E-mail: asato@md.tsukuba.ac.jp

All rights are reserved to the Japanese Circulation Society. For permissions, please e-mail: cj@j-circ.or.jp

ISSN-1346-9843 


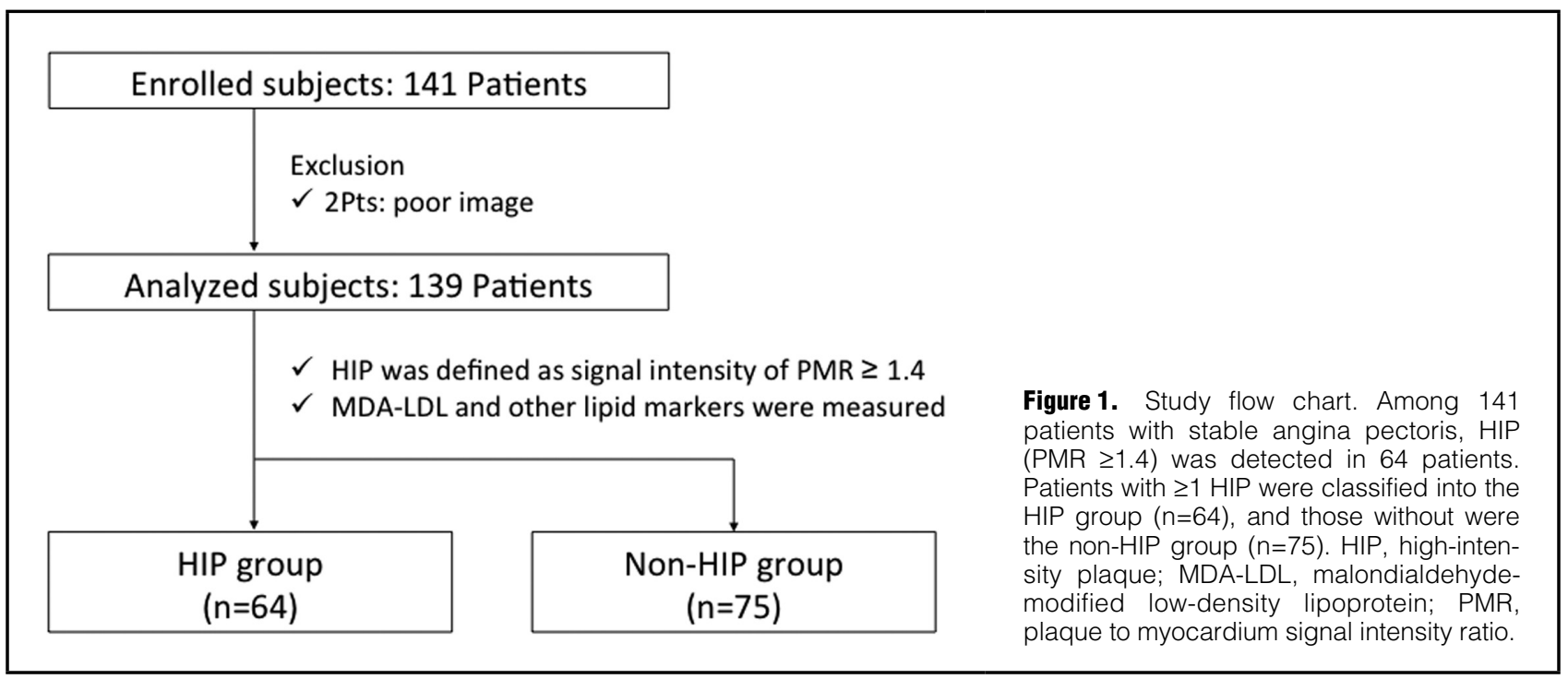

cells, and activation of pro-inflammatory molecule production in endothelial and smooth muscle cells. ${ }^{6}$ Therefore, elevated OxLDL levels could play a critical role in the transition from stable to vulnerable plaque. Malondialdehyde-modified LDL (MDA-LDL), which is known to be OxLDL, is reported to be a marker of CAD severity, plaque vulnerability, and a predictor of ACS. ${ }^{7}$ Matsuo et al reported that circulating MDA-LDL levels were associated with the presence of thin-cap fibroatheromas as assessed by optical coherence tomography. ${ }^{8}$ However, there were no studies comparing coronary HIP detected on non-contrast T1WI and circulating MDA-LDL levels for the prediction of cardiac events.

Therefore, we hypothesized that the presence of HIP might be associated with elevated serum levels of MDALDL, and the aim of this study was to investigate the additional effect of coronary HIP with MDA-LDL on cardiac events after PCI.

\section{Methods}

\section{Study Population}

Between January 2012 and August 2016, 141 patients with stable angina pectoris in whom significant coronary artery stenosis $(>70 \%)$ was diagnosed by invasive coronary angiography (CAG) were prospectively enrolled before undergoing coronary magnetic resonance imaging (MRI) with non-contrast T1WI. Of them, 2 patients were excluded for poor T1WI image quality; therefore, 139 patients were analyzed in this study (Figure 1). Patients with at least 1 HIP were classified into the HIP group. Exclusion criteria included patients with a contraindication for MRI (pacemaker or implantable cardioverter defibrillator) and chronic total occlusion. Venous blood samples were collected within the $24 \mathrm{~h}$ prior to MRI, and MDA-LDL levels and other lipid-related markers such as triglycerides, highdensity lipoprotein cholesterol (HDL-C), low-density lipoprotein cholesterol (LDL-C), lipoprotein (a), remnant-like lipoprotein particles cholesterol (RLP-C), and eicosapentaenoic acid/arachidonic acid (EPA/AA) were measured. Scheduled PCI was performed within $48 \mathrm{~h}$ after MRI using the standard technique. Cardiac events were defined as major adverse cardiac events (MACE: cardiac death, myocardial infarction (MI), and/or ischemia-driven PCI due to progressive angina pectoris). MACE was investigated during the follow-up period (5.6 \pm 1.3 years). When the patient developed any MACE, the culprit lesion was detected by CAG. This study was approved by the Institutional Review Board of the University of Tsukuba and written informed consent was given by all patients.

\section{Cardiac MRI}

All subjects were imaged on a 1.5 T MR system (Achieva, Philips Healthcare, Best, The Netherlands) using a 32-element torso/cardiac phased-array coil. The procedures used to acquire MR images in this study have been previously described. ${ }^{5}$ Briefly, following scout imaging to localize the heart and diaphragm, transaxial cine MR images were acquired to monitor the interval of minimal motion of the right coronary artery for the determination of the trigger delay of the following sequences. First, to obtain detailed information on the location of the target lesion, freebreathing, steady-state, free-precession, axial-based threedimensional (3D) whole-heart coronary MR angiographic images were obtained (repetition time, $2.5 \mathrm{~ms}$; echo time, $1.3 \mathrm{~ms}$; flip angle, $80^{\circ}$; sensitivity-encoding factor, 2.5 ; field of view, $300 \times 300 \times 120 \mathrm{~mm}$; acquisition matrix, $192 \times 191$; acquired spatial resolution, $1.56 \times 1.56 \times 2.00 \mathrm{~mm}$, reconstructed voxel size, $0.59 \times 0.59 \times 1.00 \mathrm{~mm})$. The following coronary plaque images were obtained by using a T1-weighted, inversion-recovery, and fat-suppressed 3D black-blood gradient-echo sequence with navigator-gated free-breathing and ECG-gated techniques (repetition time, $4.9 \mathrm{~ms}$; echo time, $2.3 \mathrm{~ms}$; flip angle, $15^{\circ}$; sensitivity-encoding factor, 2.0; field of view, $300 \times 300 \times 120 \mathrm{~mm}$; acquisition matrix, $208 \times 208$; acquired spatial resolution, $1.44 \times 1.42 \times 2.00 \mathrm{~mm}$, reconstructed voxel size, $0.59 \times 0.59 \times 1.00 \mathrm{~mm})$. The inversion time $(400-500 \mathrm{~ms})$ of the inversion-recovery sequence was adjusted to null the blood signal. ${ }^{9}$ The mean acquisition times for MR angiography and plaque imaging were $11 \pm 3 \mathrm{~min}$ and $16 \pm 3 \mathrm{~min}$, respectively.

\section{Plaque Analysis on Cardiac MRI}

The images were stored on an optical disk in DICOM 
A) HIP

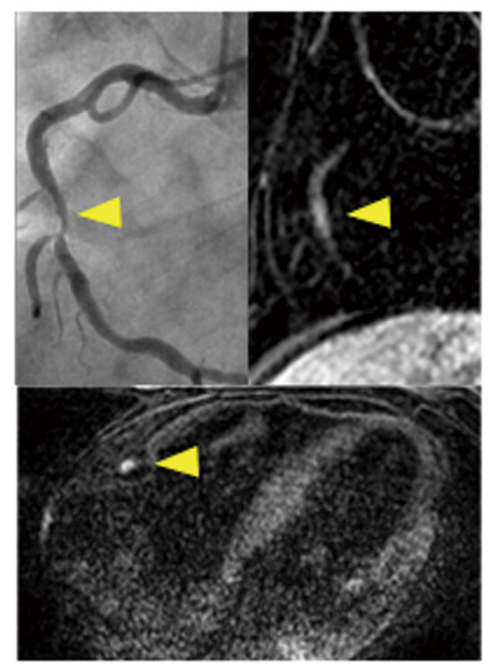

B) Non-HIP

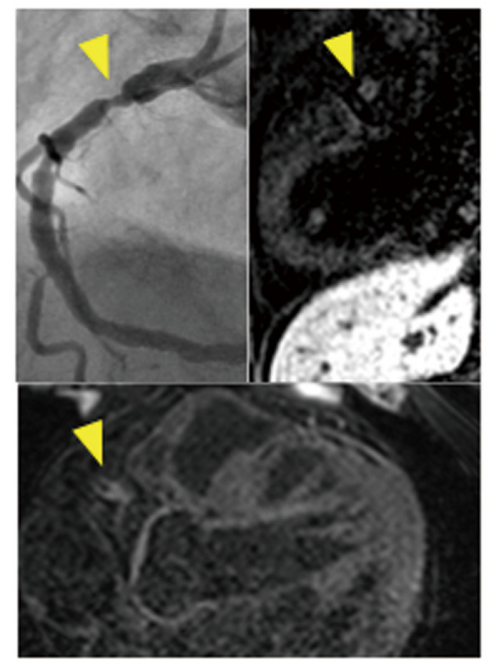

\begin{tabular}{|ll|ll|}
\hline PMR & 2.12 & PMR & 1.11 \\
\hline MDA-LDL & $170 \mathrm{U} / \mathrm{L}$ & MDA-LDL & $79 \mathrm{U} / \mathrm{L}$ \\
\hline
\end{tabular}

Figure 2. Representative cases of HIP and non-HIP. (A) A 61-year-old man presented with severe stenosis in the mid right coronary artery (RCA). T1WI revealed that the lesion was HIP (PMR=2.12). Further analysis revealed very high MDA-LDL levels (170U/L). (B) An 80-year-old man presented with significant stenosis in the proximal RCA. The lesion was non-HIP by T1WI (PMR=1.11). Further analysis revealed that his MDA-LDL levels were relatively low $(79 \mathrm{U} / \mathrm{L})$. HIP, highintensity plaque; MDA-LDL, malondialdehyde-modified low-density lipoprotein; PMR, plaque to myocardium signal intensity ratio. format. The data were analyzed off-line with the DICOM Viewer R3.0 SP3 software (Philips Healthcare). Two experienced cardiologists, who were blinded to the patient's clinical data, measured the signal intensities of coronary plaque and cardiac muscle by placing free-hand regions of interest (ROI) on a standard console of the clinical MR system and calculating the value of PMR, which was defined as the signal intensity of the coronary plaque divided by the signal intensity of cardiac muscle. The signal intensity of the myocardium was measured at a site in the left ventricle near the coronary plaque. The highest signal intensity detected in each plaque was considered as the PMR value for that plaque. A coronary plaque with a $\mathrm{PMR} \geq 1.4$ was defined as HIP, and one with a PMR $<1.4$ was defined as non-HIP (Figure 2). This definition was based on the finding of a previous study that demonstrated that a coronary plaque with a PMR $\geq 1.4$ was associated with a poor clinical prognosis. ${ }^{4}$

The location of the target lesion was determined by carefully comparing the CAG and MR angiographic images using fiduciary points such as side branches. Once the target lesion had been confirmed using coronary MR angiography, the areas corresponding to the above site on coronary T1WI were carefully matched according to the surrounding cardiac and chest wall structures. Intraclass correlation coefficients were calculated to assess intra- and interobserver agreements for PMR. The intra- and interobserver intraclass correlation coefficients were 0.91 and 0.85 , respectively.

\section{Measurement of MDA-LDL and Other Lipid-Related \\ Markers}

Venous blood samples were collected while patients were in the fasting state in the $24 \mathrm{~h}$ prior to cardiac MRI. HDLC, triglycerides, lipoprotein (a), RLP-C, and EPA/AA levels were measured by enzymatic methods. LDL-C levels were calculated by the Friedwald equation. The MDALDL levels were measured by ELISA (Sekisui Medical Co, Tokyo, Japan), as previously reported. ${ }^{10}$ Briefly, samples were diluted 2,000-fold in a dilution buffer containing $3.5 \mathrm{mM}$ sodium dodecyl sulfate. Duplicate $100-\mu \mathrm{L}$ portions of samples were added to the wells of plates coated with monoclonal antibody against MDA-LDL (ML25), then a $\beta$-galactosidase-conjugated monoclonal antibody against apoB (AB16) was added. Following incubation for $1 \mathrm{~h}$ at room temperature, o-nitrophenyl-galactopyranoside was added. We tentatively defined $1 \mathrm{U} / \mathrm{L}$ MDA-LDL as the absorbance obtained with the primary standard at a concentration of $1 \mathrm{mg} / \mathrm{L}$. A calibration curve was prepared by diluting a reference serum as a secondary standard from 300 to 9,600-fold with a dilution buffer and used to calculate the amount of MDA-LDL in the samples.

\section{Statistical Analysis}

All data are expressed as the mean \pm standard deviation, or in terms of numbers and percentages. Comparisons of continuous variables between 2 groups were made by analysis of variance. The comparisons of categorical variables between groups were performed by Fisher exact test. Logistic regression analysis was used to identify independent predictors of the presence of coronary HIP on T1WI. The univariable predictors with a $\mathrm{P}$ value $<0.1$ were entered into a multivariable model. In addition, age, sex, diabetes mellitus, and high-sensitivity C-reactive protein (hs-CRP) were forced into the multivariable model because the multivariable model should adjust for variables known to be predictive for vulnerable plaque. A multivariable Cox proportional hazard model was performed to identify the independent MACE determinants and was divided into 2 models because of collinearity between the MDA-LDL and PMR. A receiver operating characteristic (ROC) curve was constructed to identify the optimal cutoff for MDA- 


\begin{tabular}{|c|c|c|c|c|}
\hline & $\begin{array}{c}\text { All } \\
(n=139)\end{array}$ & $\begin{array}{l}\text { HIP }(+) \\
(n=64)\end{array}$ & $\begin{array}{l}\text { HIP (-) } \\
(n=75)\end{array}$ & $P$ value \\
\hline Age (years) & $68 \pm 10$ & $67 \pm 8$ & $68 \pm 11$ & 0.879 \\
\hline Sex (male) & $118(85 \%)$ & $52(81 \%)$ & $66(88 \%)$ & 0.529 \\
\hline Hypertension & $94(68 \%)$ & $43(67 \%)$ & $51(68 \%)$ & 0.816 \\
\hline Dyslipidemia & $102(73 \%)$ & $48(75 \%)$ & $54(72 \%)$ & 0.703 \\
\hline Diabetes mellitus & $54(39 \%)$ & $25(39 \%)$ & $29(39 \%)$ & 0.936 \\
\hline Smoking & 77 (55\%) & $41(64 \%)$ & $36(48 \%)$ & 0.203 \\
\hline hsCRP & $0.21 \pm 0.45$ & $0.19 \pm 0.32$ & $0.22 \pm 0.54$ & 0.733 \\
\hline \multicolumn{5}{|c|}{ Lipid profile markers (mg/dL) } \\
\hline Total cholesterol & $165 \pm 30$ & $172 \pm 35$ & $159 \pm 23$ & 0.064 \\
\hline Triglyceride & $145 \pm 76$ & $154 \pm 74$ & $137 \pm 77$ & 0.353 \\
\hline $\mathrm{HDL}$ & $46 \pm 11$ & $45 \pm 12$ & $47 \pm 10$ & 0.431 \\
\hline LDL & $96 \pm 26$ & $102 \pm 33$ & $91 \pm 17$ & 0.068 \\
\hline $\operatorname{Lp}(a)$ & $17 \pm 16$ & $19 \pm 18$ & $16 \pm 14$ & 0.306 \\
\hline RLP-C & $8 \pm 6$ & $9 \pm 5$ & $8 \pm 6$ & 0.360 \\
\hline MDA-LDL & $91 \pm 30$ & $106 \pm 30$ & $78 \pm 23$ & $<0.0001$ \\
\hline EPA/AA & $0.52 \pm 0.22$ & $0.47 \pm 0.21$ & $0.56 \pm 0.23$ & 0.109 \\
\hline Single-vessel disease & $58(42 \%)$ & $21(33 \%)$ & 37 (49\%) & 0.185 \\
\hline Multivessel disease & $81(58 \%)$ & $43(67 \%)$ & $38(51 \%)$ & 0.185 \\
\hline Multiple HIPs & $21(15 \%)$ & $21(33 \%)$ & $0(0 \%)$ & $<0.0001$ \\
\hline \multicolumn{5}{|l|}{ Medications } \\
\hline Aspirin & $139(100 \%)$ & $64(100 \%)$ & 75 (100\%) & 1.000 \\
\hline Clopidogrel & $139(100 \%)$ & $64(100 \%)$ & $75(100 \%)$ & 1.000 \\
\hline Statin & $125(90 \%)$ & $62(97 \%)$ & $63(84 \%)$ & 0.076 \\
\hline$\beta$-blocker & $89(64 \%)$ & $39(61 \%)$ & $50(67 \%)$ & 0.594 \\
\hline ACEI and/or ARB & $60(43 \%)$ & $29(45 \%)$ & $31(41 \%)$ & 0.705 \\
\hline
\end{tabular}

Values are reported as the mean \pm standard deviation or $n(\%)$. ACEI, angiotensin converting enzyme inhibitor; ARB, angiotensin receptor blocker; EPA/AA, eicosapentaenoic acid/arachidonic acid; HDL, high-density lipoprotein cholesterol; HIP, high-intensity plaque; hsCRP, high-sensitivity C-reactive protein; LDL, low-density lipoprotein cholesterol; Lp(a), lipoprotein(a); MDA-LDL, malondialdehyde-modified LDL; RLP-C, remnant-like lipoprotein particles cholesterol.

LDL to detect coronary HIP, and to compare MACE prediction. Kaplan-Meier curves were made to describe the freedom from MACE after PCI, and the log-rank test was used to identify the significant differences in unadjusted survival rate among groups. Moreover, the increased PMR discriminatory value was further examined by net reclassification improvement (NRI). Throughout the analysis, a two-sided $P$ value $<0.05$ was considered to be statistically significant. All statistical analyses were performed with JMP 11 (SAS Inc., Cary, NC, USA) or R software version 3.6.1 (R Foundation for Statistical Computing, Vienna, Austria).

\section{Results}

Association of HIP on T1 WI and Circulating MDA-LDL

The overall prevalence of HIP in patients with stable angina pectoris was $46 \%(64 / 139 ; 1$ HIP, $n=43 ; 2$ HIPs, $\mathrm{n}=21$ ). There were no significant differences in coronary risk factors and lipid profile markers except for MDALDL in the patients with and without HIP. Circulating levels of MDA-LDL $(\mathrm{P}<0.0001)$ were significantly higher in patients with HIP than in those without HIP (Table 1). Although patients with diabetes had higher MDA-LDL levels than those without diabetes, there was no significant difference $(94.0 \pm 35.1 \mathrm{U} / \mathrm{L}$ vs. $88.9 \pm 22.9 \mathrm{U} / \mathrm{L}, \mathrm{P}=0.483)$. We performed a correlated analysis between MDA-LDL and

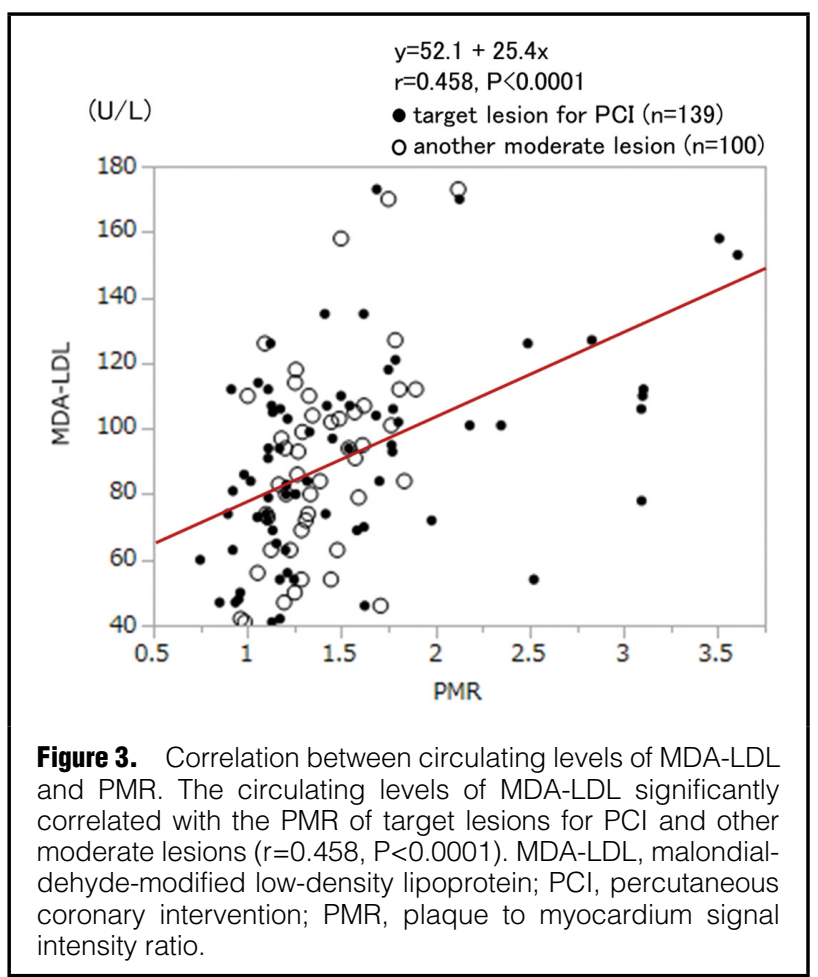




\begin{tabular}{|c|c|c|c|c|}
\hline & 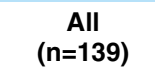 & $\begin{array}{c}\text { MACE (+) } \\
(n=21)\end{array}$ & $\begin{array}{c}\text { MACE (-) } \\
(n=118)\end{array}$ & $P$ value \\
\hline Age (years) & $68 \pm 10$ & $65 \pm 11$ & $68 \pm 9$ & 0.391 \\
\hline Sex (male) & $118(85 \%)$ & $19(90 \%)$ & $99(84 \%)$ & 0.512 \\
\hline Hypertension & $94(68 \%)$ & $15(71 \%)$ & $79(67 \%)$ & 0.715 \\
\hline Dyslipidemia & $102(73 \%)$ & $13(62 \%)$ & $89(75 \%)$ & 0.427 \\
\hline Diabetes mellitus & $54(39 \%)$ & $9(43 \%)$ & $45(38 \%)$ & 0.630 \\
\hline Smoking & $77(55 \%)$ & $17(81 \%)$ & $60(51 \%)$ & 0.047 \\
\hline hsCRP & $0.21 \pm 0.45$ & $0.35 \pm 0.50$ & $0.18 \pm 0.44$ & 0.251 \\
\hline \multicolumn{5}{|c|}{ Lipid profile markers (mg/dL) } \\
\hline Total cholesterol & $165 \pm 30$ & $179 \pm 34$ & $163 \pm 28$ & 0.092 \\
\hline Triglyceride & $145 \pm 76$ & $157 \pm 80$ & $143 \pm 75$ & 0.578 \\
\hline $\mathrm{HDL}$ & $46 \pm 11$ & $47 \pm 14$ & $46 \pm 11$ & 0.734 \\
\hline LDL & $96 \pm 26$ & $106 \pm 32$ & $94 \pm 24$ & 0.157 \\
\hline $\operatorname{Lp}(a)$ & $17 \pm 16$ & $17 \pm 21$ & $17 \pm 15$ & 0.987 \\
\hline RLP-C & $8 \pm 6$ & $9 \pm 6$ & $8 \pm 6$ & 0.660 \\
\hline MDA-LDL & $91 \pm 30$ & $115 \pm 41$ & $86 \pm 26$ & 0.003 \\
\hline EPA/AA & $0.52 \pm 0.22$ & $0.41 \pm 0.17$ & $0.53 \pm 0.22$ & 0.123 \\
\hline Single-vessel disease & $58(42 \%)$ & $4(19 \%)$ & $54(46 \%)$ & 0.073 \\
\hline Multivessel disease & $81(58 \%)$ & $17(81 \%)$ & $64(54 \%)$ & 0.073 \\
\hline Multiple HIPs & $21(15 \%)$ & $15(71 \%)$ & $6(5 \%)$ & $<0.0001$ \\
\hline PMR & $1.54 \pm 0.67$ & $2.15 \pm 0.79$ & $1.43 \pm 0.59$ & 0.0008 \\
\hline \multicolumn{5}{|l|}{ Medications } \\
\hline Aspirin & $139(100 \%)$ & $21(100 \%)$ & $118(100 \%)$ & 1.000 \\
\hline Clopidogrel & $139(100 \%)$ & $21(100 \%)$ & $118(100 \%)$ & 1.000 \\
\hline Statin & $125(90 \%)$ & $19(90 \%)$ & $106(90 \%)$ & 0.938 \\
\hline$\beta$-blocker & $89(64 \%)$ & $13(62 \%)$ & $75(63 \%)$ & 0.985 \\
\hline ACEI and/or ARB & $60(43 \%)$ & $13(62 \%)$ & $47(40 \%)$ & 0.136 \\
\hline
\end{tabular}

Values are reported as the mean \pm standard deviation or $\mathrm{n}(\%)$. MACE, major adverse cardiac events; PMR, plaque to myocardium signal intensity ratio. Other abbreviations as in Table 1.

PMR, and the circulating level of MDA-LDL showed a significant correlation with PMR $(\mathrm{r}=0.490, \mathrm{P}<0.0001)$. Of all patients, $100(72 \%)$ had non-culprit moderate coronary stenosis $(30 \%-70 \%)$. MDA-LDL levels also showed a significant correlation with the PMR of non-culprit moderate coronary stenosis $(\mathrm{r}=0.512, \mathrm{P}<0.0001)$. When these lesions were analyzed together, MDA-LDL and PMR were significantly correlated $(\mathrm{r}=0.458, \mathrm{P}<0.0001)$ (Figure 3). Furthermore, among the patients with HIP and high PMR $(\geq 1.4)$, MDA-LDL and PMR were significantly correlated $(r=0.276, P=0.046)$ (Supplementary Figure 1). In the multivariable logistic regression analysis, MDA-LDL levels were independently associated with the presence of HIP (odds ratio (OR) 1.05; 95\% confidence interval (CI), 1.02$1.08, \mathrm{P}<0.0001)$ (Supplementary Table). The prediction of circulating MDA-LDL level for coronary HIP was assessed by ROC analysis. MDA-LDL, at a cutoff of $90.4 \mathrm{U} / \mathrm{L}$, showed a sensitivity and specificity of $80 \%$ and $66 \%$, respectively (area under the curve [AUC], 0.772) (Supplementary Figure 2).

\section{Incidence of MACE After PCI in Patients With and Without HIP}

The overall incidence of MACE in patients after PCI for stable angina pectoris was $15 \%(21 / 139)$ (cardiac death, $\mathrm{n}=2$; MI, $\mathrm{n}=5$; ischemia-driven PCI due to progressive angina pectoris, $n=14)$ during the long-term follow-up. Among all 21 patients with MACE, 17 (81\%) (2 with car- diac death, 3 with MI, and 12 with ischemia-driven PCI due to progressive angina pectoris) had non-culprit moderate coronary stenosis (30-70\%). Of these 17 lesions, coronary HIPs were identified in 15 lesions, of which 13 lesions $(87 \%)$ were responsible for MI or ischemia-driven PCI with definitive diagnosis confirmed by CAG. The patients with MACE had significantly higher rates of a smoking history. Circulating MDA-LDL levels $(\mathrm{P}=0.003)$ were significantly higher in patients with MACE than in those without MACE. Furthermore, the PMR value was greater in patients with MACE than in those without MACE ( $2.15 \pm 0.79$ vs. $1.43 \pm 0.59, \mathrm{P}=0.0008)$, and the proportion of patients with multiple HIPs was higher in patients with MACE than in those without MACE (71\% vs. 5\%, $\mathrm{P}<0.0001)$ (Table 2). The incidence of MACE in patients with HIP $(27 \%)$ was significantly higher than that in patients without HIP $(5 \% ; \mathrm{P}=0.011$ by the log-rank test) (Figure 4A). In the multivariable Cox proportional hazard analysis, the MDA-LDL level and PMR were significantly associated with MACE (MDA-LDL: hazard ratio (HR) $1.03,95 \%$ CI $1.01-1.05, \mathrm{P}=0.007$; PMR: HR 2.39, 95\% CI, 1.19-4.65, $\mathrm{P}=0.016)$ (Table 3).

\section{Comparison of MACE Prediction After PCI Between MDA-LDL Level and PMR}

For MACE prediction, the C-statistic values for MDALDL, PMR, and PMR+MDA-LDL were 0.724, 0.791, 
A

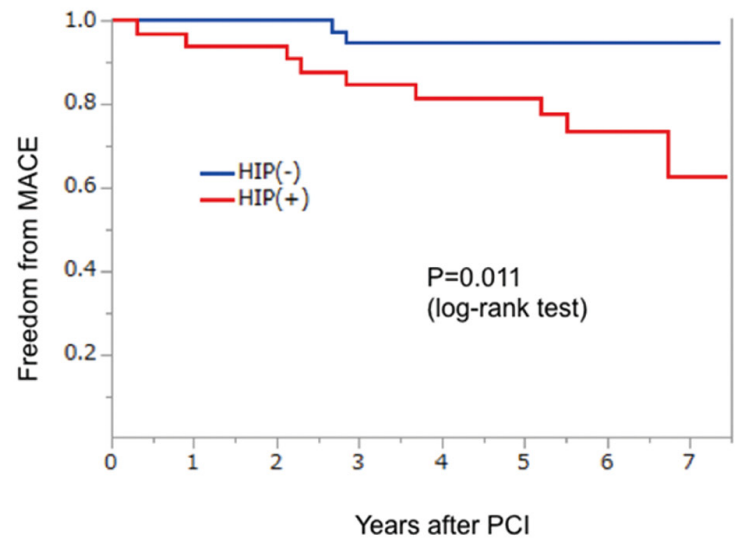

B

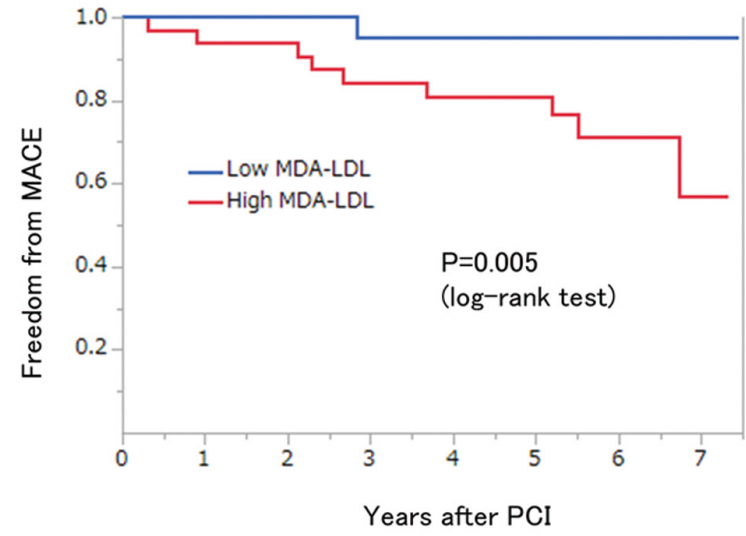

Figure 4. Kaplan-Meier curves for prediction of MACE. (A) $P=0.011$ for the HIP group vs. non-HIP group (log-rank test). (B) $\mathrm{P}=0.005$ for the high MDA-LDL group vs. Iow MDA-LDL group (log-rank test). HIP, high-intensity plaque; MACE, major adverse cardiac event; MDA-LDL, malondialdehyde-modified low-density lipoprotein; PCI, percutaneous coronary intervention.

Table 3. Univariable and Multivariable Cox Proportional Hazard Models for Predicting MACE

\begin{tabular}{|c|c|c|c|c|c|c|}
\hline \multirow{2}{*}{ Variable } & \multicolumn{2}{|c|}{ Univariable } & \multicolumn{2}{|c|}{ Multivariable: Model A $^{*}$} & \multicolumn{2}{|c|}{ Multivariable: Model $\mathrm{B}^{\dagger}$} \\
\hline & HR $(95 \% \mathrm{Cl})$ & $P$ value & HR (95\% Cl) & $P$ value & HR $(95 \% \mathrm{Cl})$ & $P$ value \\
\hline Age & $0.97(0.92-1.04)$ & 0.414 & $0.98(0.92-1.05)$ & 0.567 & $0.96(0.90-1.03)$ & 0.230 \\
\hline Male & $1.96(0.37-36.0)$ & 0.484 & $0.85(0.11-17.9)$ & 0.891 & $1.03(0.12-22.4)$ & 0.981 \\
\hline Hypertension & $1.23(0.36-5.63)$ & 0.753 & & & & \\
\hline Diabetes mellitus & $1.41(0.40-4.72)$ & 0.572 & & & & \\
\hline Smoking & $4.32(1.10-28.6)$ & 0.035 & $3.75(0.82-28.7)$ & 0.091 & $3.62(0.73-29.5)$ & 0.121 \\
\hline hsCRP & $1.40(0.45-2.64)$ & 0.468 & & & & \\
\hline LDL & $1.02(0.99-1.04)$ & 0.123 & & & & \\
\hline MDA-LDL & $1.03(1.01-1.05)$ & 0.002 & $1.03(1.01-1.05)$ & 0.007 & - & - \\
\hline PMR & $2.59(1.33-4.88)$ & 0.006 & - & - & $2.39(1.19-4.65)$ & 0.016 \\
\hline
\end{tabular}

*Variables analyzed in model A: age, sex, smoking, and MDA-LDL. †Variables analyzed in model B: age, sex, smoking, and PMR. Multivariable Cox proportional hazard model was divided into 2 models because of collinearity between MDA-LDL and PMR. Cl, confidence interval; HR, hazard ratio. Other abbreviations as in Tables 1,2.

\begin{tabular}{|c|c|c|c|}
\hline & C-statistic & NRI (95\% Cl) & $P$ value \\
\hline MDA-LDL & 0.724 & & \\
\hline PMR & 0.791 & & \\
\hline PMR+MDA-LDL & 0.800 & $0.78(0.17-1.39)$ & 0.012 \\
\hline
\end{tabular}

$\mathrm{NRI}$, net reclassification index. Other abbreviations as in Tables 1-3.

and 0.800 , respectively (Table 4). ROC analysis showed that MDA-LDL, at a cutoff of 95.0 U/L, showed a sensitivity and specificity of $81 \%$ and $62 \%$, respectively. In addition, the incidence of MACE in patients with high ( $\geq 95 \mathrm{U} / \mathrm{L})$ MDA-LDL (28\%) was significantly higher than that in patients with low (<95U/L) MDA-LDL $(5 \%$; $\mathrm{P}=0.005$ by the log-rank test) (Figure 4B). PMR, at a cutoff value of 1.68 , showed a sensitivity and specificity of $72 \%$ and $79 \%$, respectively. Compared with MDA-LDL alone, the addition of PMR to MDA-LDL increased the NRI by $0.78(\mathrm{P}=0.012)$.

\section{Discussion}

The major findings of the present study were as follows: (1) circulating levels of MDA-LDL significantly correlated 
with PMR; (2) the incidence of MACE in patients with HIP was significantly higher than that in patients without HIP. In addition, most patients with MACE had nonculprit moderate coronary stenosis, and HIP was detected by CMR; (3) MDA-LDL levels and PMR were significantly associated with MACE. Compared with MDALDL alone, the addition of PMR to MDA-LDL increased the NRI by $0.78(\mathrm{P}=0.012)$.

To the best of our knowledge, this is the first study to assess the association between coronary HIP, circulating level of MDA-LDL, and cardiac events after PCI.

\section{Pathological Association Between HIP and MDA-LDL}

Intraplaque hemorrhage (IPH) often occurs within lipidrich necrotic cores, and the methemoglobin in IPH leads to a significant shortening in T1-relaxation time. ${ }^{11}$ Therefore, detecting HIP on T1WI shows promise for the in vivo identification of vulnerable plaques associated with thrombus or IPH. IPH has been associated with more rapid growth of the lipid core and accelerated enlargement of the plaque, resulting in luminal narrowing. ${ }^{\mathbf{1 2}}$

Atherosclerosis is considered to be a chronic inflammatory disease, and inflammation and oxidative stress appear to be closely linked. ${ }^{\mathbf{1 3}}$ The oxidative modification hypothesis of atherogenesis suggests that the most significant event in early lesion formation is lipid oxidation. Circulating concentrations of MDA-LDL are thought to directly reflect oxidative stress in vivo. We reported that MDALDL levels were independently associated with the presence of HIP. Amaki et al reported that MDA-LDL was an independent risk factor of CAD and that MDA-LDL concentrations at a cutoff of $85.6 \mathrm{U} / \mathrm{L}$ showed a sensitivity and specificity of $64 \%$ and $65 \%$, respectively. ${ }^{14}$ In this study, MDA-LDL to predict coronary HIP, at a cutoff of $90.4 \mathrm{U} / \mathrm{L}$, showed a sensitivity and specificity of $80 \%$ and $66 \%$, respectively.

\section{Comparison of the Predictive Ability of Coronary HIP and MDA-LDL for MACE}

Although several randomized studies have demonstrated the benefits of statins in reducing both death and the incidence of ACS, ${ }^{15}$ some high-risk patients develop recurrent cardiac events despite their LDL-C level being controlled by statin treatment. ${ }^{16}$ Ito et al suggested that MDA-LDL was associated with future cardiac events in patients with stable angina who underwent lipid-lowering therapy after PCI, among $94 \%$ of patients who underwent statin therapy. ${ }^{17}$ A previous study reported that, over a 3-year period, the incidence of cardiac death, MI and/or hospitalization for heart failure was significantly higher in the high MDALDL patients than in the low MDA-LDL patients. ${ }^{7}$

In addition, we demonstrated that most patients with MACE had non-culprit moderate coronary stenosis in which HIP was detected by CMR. Stone et al investigated 697 patients with ACS for 3.4 years, and showed that MACE were equally attributable to recurrence at the site of culprit (12.9\%) and non-culprit lesions (11.6\%) and that most non-culprit lesions responsible for follow-up events were angiographically mild at baseline $(32.3 \pm 20.6 \%) .{ }^{18} \mathrm{In}$ the present study, of 21 patients with MACE after PCI, 17 had non-culprit moderate stenosis identified by baseline CAG. Among the 17 lesions, coronary HIPs were detected in 15, of which 13 lesions (87\%) were responsible for MI or ischemia-driven PCI with definitive diagnosis confirmed by CAG. This would suggest that careful follow-up is required when coronary HIP is identified by CMR, even in moderately stenotic lesions. We showed that the MDA-LDL level, which indicates coronary plaque instability, was higher in patients with HIP and high PMR. The measurement of MDA-LDL levels may be helpful to predict high PMR in patients with HIP. This result has increased the importance for the MRI index PMR which is important as an index to predict MACE after PCI. Furthermore, we demonstrated that the addition of PMR to MDA-LDL increased the NRI by $0.78(\mathrm{P}=0.012)$. DemLer et al recommended estimating formula-based standard errors and confidence intervals of continuous NRI when the significance of predictor variables is strong enough. ${ }^{19}$ On the other hand, some suggest that the NRI is not a proper measure of performance improvement..$^{20}$ Therefore, careful evaluation should be done when interpreting the NRI statistic.

\section{Study Limitations}

First, the main limitation was the lack of standardization and the quantitative nature of HIP diagnosis by MRI, which is based on the relative 'eye balling' nature of this diagnosis and manual ROI tracings. HIP can be easily identified and the ROI can be drawn around it. However, it seems challenging to identify non-HIP and draw an ROI around it. Therefore, we identified the target plaque by carefully comparing the $\mathrm{CAG}$ and $\mathrm{MR}$ angiographic images using fiduciary points. Second, the study was conducted at a single center and the study population was relatively small. MACE occurred in 21 of 139 patients. However, two-thirds (14/21) of the MACE were late revascularizations. The value of HIP on CMR was limited for the prediction of cardiac death and MI. Third, histological validation of coronary plaque with HIP was lacking because it is difficult to obtain coronary plaque specimens from human coronary arteries in vivo, and as such, the precise characterization of coronary HIP remains unknown. In addition to T1WI, simultaneous multiple MRI sequences (e.g., T2-weighted, proton density-weighted, or time-offlight sequence) would be potentially useful for more precise coronary plaque characterization with a comprehensive/ holistic approach. Fourth, the NRI statistic is controversial. Finally, in this study, most patients underwent statin therapy prior to blood sampling, and as such, their lipid profiles were modified. Because Asians have a lighter body weight than Caucasians, $5-10 \mathrm{mg} /$ day of atorvastatin and $2.5-5 \mathrm{mg} /$ day of rosuvastatin are the standard doses in Japan and atorvastatin $20 \mathrm{mg} /$ day and rosuvastatin $20 \mathrm{mg} /$ day are the approved highest doses. In this study, $90 \%$ patients were prescribed rosuvastatin 2.5 or $5 \mathrm{mg}$. Komukai et al demonstrated that serum MDA-LDL levels were significantly decreased in the $20 \mathrm{mg}$ /day group but not in the group receiving $5 \mathrm{mg} /$ day of atorvastatin. ${ }^{21}$ Further studies with a larger sample size and the participation of many hospitals will assist in confirming the validity of the results of the current study.

\section{Conclusions}

MDA-LDL levels might be associated with the presence of HIP in patients with CAD. Furthermore, adding PMR to the MDA-LDL levels markedly improved the prediction of MACE subsequent to PCI.

\section{Acknowledgments}

We thank Editage (www.editage.jp) for English language editing. 


\section{Ethics Approval and Consent to Participate}

This study was approved by the Institutional Review Board of the University of Tsukuba and written informed consent was given by all patients.

\section{Consent for Publication}

Not applicable; non-identifiable data only included.

\section{Availability of Data and Materials}

The datasets used and/or analyzed during the current study are available from the corresponding author on reasonable request.

\section{Competing Interests}

All authors declare that they have no competing interests.

\section{Funding}

None.

\section{Authors' Contributions}

D.H. and A.S. contributed to the conception and design of this work, drafting of the article, and writing and revising the manuscript. T.H., S.S., and H.W. contributed for analysis and interpretation of circulating levels of MDA-LDL and MRI data. M.I. contributed to critical revision of the manuscript for important intellectual content. All authors have read and approved the manuscript.

\section{References}

1. Libby P. Inflammation in atherosclerosis. Nature 2002; 420: 868-874.

2. Yuan C, Mitsumori LM, Ferguson MS, Polissar NL, Echelard $\mathrm{D}$, Ortiz G, et al. In vivo accuracy of multispectral magnetic resonance imaging for identifying lipid-rich necrotic cores and intraplaque hemorrhage in advanced human carotid plaques. Circulation 2001; 104: 2051-2056.

3. Kawasaki T, Koga S, Koga N, Noguchi T, Tanaka H, Koga H, et al. Characterization of hyperintense plaque with noncontrast $\mathrm{T}(1)$-weighted cardiac magnetic resonance coronary plaque imaging: Comparison with multislice computed tomography and intravascular ultrasound. JACC Cardiovasc Imaging 2009; 2: $720-728$.

4. Noguchi T, Kawasaki T, Tanaka A, Yasuda S, Goto Y, Ishihara $\mathrm{M}$, et al. High-intensity signals in coronary plaques on noncontrast T1-weighted magnetic resonance imaging as a novel determinant of coronary events. J Am Coll Cardiol 2014; 63: 989-999.

5. Hoshi T, Sato A, Akiyama D, Hiraya D, Sakai S, Shindo M, et al. Coronary high-intensity plaque on T1-weighted magnetic resonance imaging and its association with myocardial injury after percutaneous coronary intervention. Eur Heart J 2015; 36: $1913-1922$.

6. Steinberg D. Low density lipoprotein oxidation and its pathobiological significance. J Biol Chem 1997; 272: 20963-20966.

7. Ikenaga $\mathrm{H}$, Kurisu S, Kono S, Sumimoto Y, Watanabe N, Shimonaga T, et al. Impact of malondialdehyde-modified lowdensity lipoprotein on tissue characteristics in patients with stable coronary artery disease. Circ J 2016; 80: $2173-2182$.

8. Matsuo Y, Kubo T, Okumoto Y, Ishibashi K, Komukai K,
Tanimoto T, et al. Circulating malondialdehyde-modified lowdensity lipoprotein levels are associated with the presence of thin-cap fibroatheromas determined by optical coherence tomography in cornary artery disease. Eur Heart J Cardiovasc Imaging 2013; 14: $43-50$.

9. Engel LC, Landmesser U, Abdelwahed YS, Jaguszewski M, Gigengack K, Wurster TH, et al. Comprehensive multimodality characterization of hemodynamically significant and non-significant coronary lesions using invasive and noninvasive measures. PLoS One 2020; 15: e0228292.

10. Kotani K, Maekawa M, Kanno T, Kondo A, Toda N, Manabe M. Distribution of immunoreactive malondialdehyde-modified low-density lipoprotein in human serum. Biochim Biophys Acta 1994; 1215: 121-125.

11. Moody AR, Murphy RE, Morgan PS, Martel AL, Delay GS, Allder S, et al. Characterization of complicated carotid plaque with magnetic resonance direct thrombus imaging in patients with cerebral ischemia. Circulation 2003; 107: 3047-3052.

12. Takaya N, Yuan C, Chu B, Saam T, Polissar NL, Jarvik GP, et al. Presence of intraplaque hemorrhage stimulates progression of carotid atherosclerotic plaques: A high-resolution magnetic resonance imaging study. Circulation 2005; 111: 2768-2775.

13. Ross R. Mechanisms of disease: Atherosclerosis: An inflammatory disease. N Engl J Med 1999; 340: 115-126.

14. Amaki T, Suzuki T, Nakamura F, Hayashi D, Imai Y, Morita $\mathrm{H}$, et al. Circulating malondialdehyde modified LDL is a biochemical risk marker for coronary artery disease. Heart 2004; 90: $1211-1213$.

15. Weintraub WS, Daniels SR, Burke LE, Franklin BA, Goff DC $\mathrm{Jr}$, Hayman LL, et al. Value of primordial and primary prevention for cardiovascular disease: A policy statement from the American Heart Association. Circulation 2011; 124: 967-990.

16. LaRosa JC, Grundy SM, Waters DD, Shear C, Barter P, Fruchart JC, et al. Intensive lipid lowering with atorvastatin in patients with stable coronary disease. $N$ Engl J Med 2005; 352: $1425-1435$.

17. Ito T, Fujita H, Tani T, Ohte N. Malondialdehyde-modified low-density lipoprotein is a predictor of cardiac events in patients with stable angina on lipid-lowering therapy after percutaneous coronary intervention using drug-eluting stent. Atherosclerosis 2015; 239: 311-317.

18. Stone GW, Maehara A, Lansky AJ, de Bruyne B, Cristea E, Mintz GS, et al; PROSPECT Investigators. A prospective natural-history study of coronary atherosclerosis. N Engl J Med 2011; 364: $226-235$.

19. DemLer OV, Pencina MJ, Cook NR, D'Agostino RB Sr. Asymptotic distribution of $\triangle A U C$, NRIs, and IDI based on theory of U-statistics. Stat Med 2017; 20: 3334-3360.

20. Pepe MS, Fan J, Feng Z, Gerds T, Hilden J. The net reclassification index (NRI): A misleading measure of prediction improvement even with independent test data sets. Stat Biosci 2015; 7: $282-295$.

21. Komukai K, Kubo T, Kitabata H, Matsuo Y, Ozaki Y, Takarada S, et al. Effect of atorvastatin therapy on fibrous cap thickness in coronary atherosclerotic plaque as assessed by optical coherence tomography: The EASY-FIT study. J Am Coll Cardiol 2014; 64: 2207-2217.

\section{Supplementary Files}

Please find supplementary file(s);

http://dx.doi.org/10.1253/circj.CJ-21-0220 